\title{
PROGRAMA DE FORTALECIMIENTO A DESTINOS TURÍSTICOS EMERGENTES: UN ANÁLISIS DESDE LA VISIÓN DEL DESARROLLO ENDÓGENO
}

\author{
Autor \\ Claudia Toselli \\ Licenciada en Turismo \\ Especialista en Turismo Cultural \\ Vicerrectorado de Investigación y Desarrollo \\ Instituto de Medio Ambiente y Ecología \\ Universidad del Salvador (Argentina) \\ claudia.toselli@salvador.edu.ar
}

\section{RESUMEN}

El objetivo de este artículo es exponer de manera objetiva un programa aplicado a nivel nacional en Argentina que tiene por objetivo promover e impulsar destinos turísticos emergentes. El programa al cual se hará referencia en concreto es el PROFODE - Programa de Fortalecimiento y Estimulo a Destinos Turísticos emergentes - implementado actualmente por la Secretaría de Turismo de la Nación Argentina (SECTUR) a través de la Dirección de Desarrollo de la Oferta que depende de la Dirección Nacional de Desarrollo Turístico. Este programa promueve el desarrollo de destinos turísticos que, por sus características naturales y/o culturales, presentan potencialidad para atraer visitantes, pero que no aún han alcanzado este objetivo debido a problemas de competitividad.

Sobre esta base, se analizará críticamente este programa siguiendo algunos lineamientos propuesto por el enfoque del desarrollo endógeno en base a ciertos autores reconocidos en la materia.

Palabras claves: Desarrollo endógeno Destinos turísticos, 


\title{
STRENGTHENING PROGRAM FOR EMERGINF TOURIST DESTINATIONS: AN ANALISYS FROM THE ENDOGENOUS DEVELOPMENT VIEW
}

\author{
Autor \\ Claudia Toselli \\ Licenciada en Turismo \\ Especialista en Turismo Cultural \\ Vicerrectorado de Investigación y Desarrollo \\ Instituto de Medio Ambiente y Ecología \\ Universidad del Salvador (Argentina) \\ claudia.toselli@salvador.edu.ar
}

\section{ABSTRACT}

The purpose of this paper is to present in an objective manner a program implemented in Argentina which aims to promote andlaunch emerging tourist destinations. The program, called PROFODE - Program for strengthening and encouraging emerging tourist destinations - is currently being implemented by the National Area of Tourism Development, which depends on the National Tourism Department of Argentina.

This program promotes the development of tourist destinations which, because of their natural or cultural features, have possibilities to attract tourists, but have not yet achieved this goal due to competitiveness issues. On this subject, the PROFODE will be critically examined, following some guidelines proposed by the endogenous development approach, based on renowned authors in this field.

Key words: Endogenous development, Tourist destinations 


\section{INTRODUCCIÓN}

El presente trabajo tiene por finalidad exponer objetivamente la metodología y acciones del PROFODE - Programa de Fortalecimiento y Estímulo a Destinos Turísticos Emergentes - implementado actualmente por la Secretaría de Turismo de la Nación Argentina (SECTUR).

Sobre la base de esta información, y en la perspectiva del desarrollo endógeno propuesta por autores como Alburquerque, Vázquez Barquero, Boisier, Arocena, Madoery, entre otros, se realizará un análisis del mismo, tratando de arribar a algunas consideraciones que permitan establecer una aproximación entre desarrollo endógeno y turismo.

Es importante anticipar aquí que este trabajo entenderá por desarrollo al "conjunto de capacidades generadas endógenamente, ligadas a la calidad de los recursos humanos, la capacidad organizativa y de articulación público-privada, la innovación y emprenditorialidad de los agentes locales, la capacidad institucional territorial, donde los impulsos exógenos se incorporan a un territorio organizado con una estructura de relaciones establecidas y consolidadas" (Madoery, 2001).

Asimismo, tal como lo postula la Organización Internacional del Trabajo (OIT, Programa Delnet, 2009), “uno de los objetivos principales de la aplicación del modelo de desarrollo endógeno es, por un lado, aprovechar las energías, competencias y recursos locales; y por otro, fomentar y establecer una sociedad capaz de contener en sí misma los modos y medios de producción necesarios para cubrir la necesidades de las personas que la integran, mediante la implementación de una serie de estrategias que influyan en lo cultural, lo económico, lo educacional y el uso e invención de tecnología”.

\section{El Programa de Fortalecimiento y Estímulo a Destinos Turísticos Emergentes}

El PROFODE - Programa de Fortalecimiento y Estímulo a Destinos Turísticos Emergentes - es un programa que operativiza la Dirección de Desarrollo de la Oferta, la cual depende de la Dirección Nacional de Desarrollo Turístico, Secretaría de Turismo de la Nación, Argentina (SECTUR). El mismo tiene sus inicios en el año 2004 y se continúa implementado en la actualidad. 
Su objetivo central es disparar, mediante acciones de fortalecimiento, procesos de desarrollo en los destinos emergentes, en el marco de una estrategia de redireccionamiento de los flujos turísticos para el crecimiento equilibrado de la actividad en el territorio nacional. Este programa se ejecuta en coordinación con otros subprogramas de la Secretaría de Turismo de la Nación, y se enmarca a su vez dentro del Plan Federal Estratégico de Turismo Sustentable 2016 (PFETS), este programa sido elaborado por la Secretaría de Turismo de la Nación en conjunto con el Consejo Federal de Inversiones, con la participación de más de 400 actores de todo el país. El objetivo del Plan es constituirse en el proceso orientador y articulador que encamine todos los esfuerzos hacia un modelo concertado de desarrollo turístico sustentable para el país. El mismo tiene un horizonte temporal a 10 años y ha definido, una visión común, además de un Mapa Federal de Oportunidades, sobre el que se ha acordado un compromiso de desarrollo hasta 2016. Este plan rige actualmente a nivel nacional en materia turística

En este punto es importante mencionar que la SECTUR entiende por destino emergente aquellos sitios que por sus características naturales y/o culturales presentan potencialidad para atraer turistas tanto nacionales como internacionales, pero que no han alcanzado aún este objetivo debido a problemas de competitividad, o bien a que la oferta turística carece de una puesta en valor adecuada o está desarticulada.

\section{Ejes rectores y objetivos del PROFODE}

El PROFODE tiene sus ejes rectores focalizados en la construcción de un modelo local basado en: a) la inclusión social y el desarrollo sustentable; b) el afianzamiento de la identidad local y la interculturalidad; c) el respeto por el patrimonio natural y cultural; d) la mejora de la calidad de vida de la población local.

Los objetivos del programa se articulan en cuatro grandes módulos, dentro de cada uno de los cuales se proponen una serie de acciones como se puede observar en el Cuadro $\mathrm{N}^{\mathrm{o}} 1$ a continuación: 


\section{Cuadro No 1}

\section{Objetivos y acciones del PROFODE}

\begin{tabular}{|c|c|}
\hline Objetivos & Acciones \\
\hline $\begin{array}{l}\text { Fortalecimiento } \\
\text { Institucional del } \\
\text { Sistema Turístico }\end{array}$ & $\begin{array}{l}\text { - } \quad \text { Trabajar en conjunto con los actores partícipes de la actividad } \\
\text { como así también con las comunidades anfitrionas. } \\
\text { Realizar acciones de capacitación para la mejora de la gestión } \\
\text { pública y privada en turismo. } \\
\text { - Facilitar relaciones institucionales entre el sector privado, } \\
\text { público y otros actores relevantes para el desarrollo de proyectos } \\
\text { turísticos. } \\
\text { Promover la modalidad de turismo comunitario y propiciar } \\
\text { el empoderamiento y gestión de la actividad por parte de los } \\
\text { agentes locales. }\end{array}$ \\
\hline $\begin{array}{l}\text { Fortalecimiento de } \\
\text { microemprendedores }\end{array}$ & $\begin{array}{l}\text { - Asesorar en la formulación de proyectos de inversión. } \\
\text { Promover el desarrollo de herramientas asociativas como } \\
\text { incubadoras y, en un estadio superior, agencias de desarrollo } \\
\text { turístico; que consoliden la oferta y contribuyan con el } \\
\text { fortalecimiento del emprendedorismo nacional y del tejido } \\
\text { empresarial local. } \\
\text { Revitalizar los trabajos de planificación turística y proyectos } \\
\text { existentes en el haber de las comunidades de los destinos y/o } \\
\text { microregiones. } \\
\text { Seleccionar proyectos turísticos viables y sostenibles mediante } \\
\text { metodologías participativas con la comunidad. }\end{array}$ \\
\hline Sistema de Soporte & $\begin{array}{l}\text { - } \quad \text { Priorizar y promover las inversiones públicas y privadas } \\
\text { necesarias para adecuar la infraestructura y equipamiento a los } \\
\text { requerimientos de la demanda. } \\
\text { Mejorar la oferta a partir de la concertación de la inversión } \\
\text { pública y la promoción de la inversión privada. }\end{array}$ \\
\hline $\begin{array}{l}\text { Desarrollo, Marketing } \\
\text { y Promoción de } \\
\text { productos }\end{array}$ & $\begin{array}{l}\text { - Articular de modo concertado y continuo los procesos de } \\
\text { desarrollo de productos en correspondencia con los espacios } \\
\text { turísticos y con los segmentos preidentificados de la demanda } \\
\text { turística. } \\
\text { Estimular la competitividad turística de los destinos emergentes, } \\
\text { potenciando el desarrollo regional. }\end{array}$ \\
\hline
\end{tabular}

Fuente: SECTUR (2009). Programa de Fortalecimiento y Estímulo a Destinos Turísticos Emergentes 


\section{Metodología de implementación del PROFODE}

Anualmente el PROFODE realiza una convocatoria dirigida a las provincias para que soliciten la aplicación del programa en aquellos destinos que correspondan al Tiempo 1, (El PFETS se ha proyectado en dos etapas: los espacios prioritarios del Tiempo 1 (2006-2011), y los del tiempo 2 (2011-2016). Para ello se debe completar una ficha de postulación, la cual es evaluada por el equipo técnico de la SECTUR, y se seleccionan aquellos destinos que reúnan las mejores condiciones para lograr una intervención exitosa del programa.

Cabe destacar aquí que en esta selección se priorizan aquellos destinos integrados por dos o más municipios asociados.

Para ello, es necesaria también la identificación y colaboración de un facilitador local, que es el nexo entre la provincia y la SECTUR durante la implementación del programa en el territorio.

En cuanto a la metodología de trabajo se realiza la elaboración de un diagnóstico en forma participativa involucrando a los diferentes actores tanto públicos como privados implicados en el sistema turístico del destino en cuestión. Asimismo, "la propuesta de trabajo propone involucrar también a distintos organismos del sector público con el objeto de realizar un abordaje integral del territorio, generando sinergias desde la especificidad de cada uno y optimizando así los recursos humanos y financieros con los que se cuenta" (SECTUR, Boletín Técnico, 07/10/2007).

En particular, un equipo conformado por técnicos de la SECTUR se traslada a las localidades implicadas y mantiene instancias grupales e individuales de intercambio sobre la visión local del desarrollo turístico en el destino. Las acciones de fortalecimiento que se definen en cada implementación son consensuadas previamente con los gobiernos locales.

Es importante aclarar que el programa trabaja el desarrollo de destinos con demanda de turismo receptivo, y también destinos con demanda de turismo interno.

De manera sintetizada, el esquema de trabajo que se propone por etapas es el siguiente: 
- Etapa Preparatoria, en la cual se formalizan los convenios de compromiso con las autoridades provinciales y/o municipales, quienes designarán un facilitador local. También se articula la organización de las actividades con el/los municipio/s y actores locales involucrados. Luego los organismos nacionales y federales elaboran un análisis de situación del destino y se convalida la articulación del PROFODE con la estrategia de desarrollo turístico viable para la zona con las autoridades provinciales y/o municipales y actores locales claves.

- Etapa de Elaboración de Diagnóstico, donde se realiza una misión exploratoria (trabajo de campo) en las áreas o corredores involucrados en el programa. Posteriormente se dialoga con las comunidades locales con el objetivo de tomar conocimiento del estado de situación del desarrollo de la actividad turística y su cadena de valor. En cuanto a la metodología de trabajo se realiza la elaboración de los diagnósticos de forma participativa involucrando a los diferentes actores, tanto públicos como privados, que tengan incidencia en el espacio turístico a trabajar.

Un equipo con representantes de los organismos involucrados en cada caso se traslada a la/s localidad/es implicadas, y mantiene instancias grupales e individuales que tienen por objeto construir la visión colectiva sobre cómo funciona la cadena de valor turística.

- Etapa de Ejecución de Acciones Específicas, dondea partir del diagnóstico efectuado, se confecciona un informe en el cual se detallan las acciones de fortalecimiento diseñadas con el propósito de contribuir a la mejora de la competitividad turística de los espacios abordados.

Por último, es importante aclarar que durante la implementación del programa, se realiza un monitoreo y evaluación de las distintas etapas, donde se considera la recolección y sistematización de datos cuantitativos y cualitativos que aporten a evaluar la satisfacción de los actores principales del destino en relación al programa y sus resultados, a partir de los cuales se realizarán sugerencias y correcciones necesarias al mismo.

Sitios de implementación del PROFODE hasta el presente

La primera experiencia del programa que se realizó fue en la Quebrada de Humahuaca, provincia de Jujuy, durante el año 2004 y 2005. Luego, durante los años 2006 y 2007, el programa se implementó en el Área Ischigualasto - Talampaya, provincia de San Juan y La Rioja. 
Posteriormente los destinos en los que se siguió aplicando fueron:

- Área Corazón de la Isla (provincia de Tierra del Fuego).

- Zona de influencia de la Puerta Corrientes - Resistencia, Isla del Cerrito (provincia de Chaco).

- Área Mar Chiquita (provincia de Córdoba).

- Corredor de la Puna (provincia de Salta).

- Corredor Neuquén Norte (provincia de Neuquén).

En la convocatoria realizada en 2008 fueron seleccionados como destinos de aplicación el Corredor de la Puna (Salta), el Corredor Neuquén Norte, y el Corredor Ruta Nacional No 40 (Chubut).

\section{Análisis del PROFODE desde el enfoque del desarrollo endógeno}

Albuquerque (1994) propone una metodología que incluye diferente aspectos a considerar en el análisis de las potencialidades de desarrollo económico local, los cuales se considerarán para analizar el PROFODE. Estos aspectos incluyen:

- Los objetivos del desarrollo económico local.

- Los recursos disponibles: humanos, físicos, técnicos, económicos, financieros, sociales, culturales, ambientales.

- Los agentes de desarrollo: administraciones públicas territoriales, agencias de desarrollo local, empresas locales, universidades, centros de investigación y desarrollo (I+D), entidades de capacitación, organizaciones no gubernamentales, entre otros.

- El proceso de planificación del desarrollo económico local.

Sobre esta base, y siguiendo la línea metodológica propuesta por este autor, surgen las siguientes consideraciones: 
I- En cuanto a los objetivos del desarrollo económico local, el PROFODE contribuye a la promoción del empleo o al mejoramiento del empleo existente, y en consecuencia contribuye a elevar la calidad de vida de los residentes del destino y promover la equidad social, por ejemplo a través de la promoción de emprendedores. También contribuye a la transformación del sistema productivo local, en particular, a través de la diversificación de actividades, como por ejemplo el turismo, en una visión de uso sostenible de los recursos naturales locales.

II- Con respecto a los recursos disponibles, tal como establece Albuquerque (1994) "la existencia de recursos en una determinada área no es condición suficiente para originar un proceso de desarrollo, ya que dichos recursos deben utilizarse de modo adecuado y dentro de una estrategia coherente y sostenible ambientalmente”.

Siguiendo esta línea, se observa que el PROFODE cada año realiza una convocatoria anual, y a partir de la presentación que hace cada municipio a través del gobierno provincial, se analizan los recursos disponibles que los destinos poseen. La enumeración de recursos se realizó siguiendo la clasificación propuesta por Albuquerque (1994) en comparación con el formulario "Ficha de postulación PROFODE - Convocatoria a Destinos 2008”, que anualmente lanza la SECTUR para realizar la convocatoria anual a los destinos que deseen postularse. A partir del análisis de los recursos físicos, y siguiendo la metodología de este mismo autor, se observa que para la preselección de los destinos que van a ingresar al programa, se toman en cuenta los siguientes aspectos: situación geográfica, recursos naturales disponibles, características ambientales, volumen y calidad de las infraestructuras básicas, vías de comunicación y acceso, transportes, telecomunicaciones, abastecimiento de agua y energía, saneamiento, alcantarillado, salud y sanidad, cultura, deporte y turismo, recogida de basura, tratamiento y reciclaje de residuos sólidos, tratamiento de aguas residuales, protección del medio ambiente local entre otros.

Dentro de los recursos físicos también se consideran los recursos intangibles, entre ellos, se analiza la cualificación de los recursos humanos, la calidad de los servicios colectivos y equipamientos urbanos, la seguridad ciudadana, la ausencia de contaminación ambiental, entre otros.

En cuanto a los recursos humanos, Alburquerque (1994) establece que "la formación para el desarrollo local debe ser una acción vinculada a un proyecto colectivo territorial en el que las actividades de formación deben basarse fundamentalmente en las necesidades y demandas existentes en el territorio". En este sentido vemos que en el PROFODE se identifican las capacidades actuales como así también los organismos que capacitan en materia de turismo, y a partir del diagnóstico que se realiza con la comunidad se determinan las necesidades en materia de formación, para dejar instaladas nuevas capacidades. 
Con relación a los recursos económicos y financieros, se trata de conocer el tejido de empresas - en general pymes o emprendimientos locales -, como así también el estado de situación del desarrollo de la actividad turística en general y su cadena de valor.

III- En cuanto a los agentes del desarrollo local, según Albuquerque (1994) "el diseño de programas de desarrollo local requiere coordinación entre los diferentes niveles de las Administraciones Públicas y, sobre todo, el acuerdo y participación de los agentes sociales”. Por lo tanto, el PROFODE es un programa que parte de la administración del Estado Central, y articula con los estados provinciales y municipales. Asimismo, involucra el sector privado, organizaciones de la sociedad civil e instituciones educativas. En este contexto, se puede visualizar que a partir de una política a nivel general, se va articulando de manera tal, que cada localidad, partiendo de su propia iniciativa, analiza las posibilidades que el turismo le ofrece como motor de desarrollo, y cada una de estas propuestas municipales, con el acuerdo y apoyo de los respectivos gobiernos provinciales, confluye en el PROFODE, y finalmente en el Plan Federal Estratégico de Turismo Sustentable (PFETS) de manera coordinada y sinérgica.

Por lo tanto, se podría afirmar que el PROFODE "posibilita mayor capacidad de autogobierno a las autoridades territoriales y refuerza el despliegue de los valores socioculturales locales. De este modo se facilita la definición de una estrategia de desarrollo endógeno más adaptada a los problemas, recursos e idiosincrasia de cada territorio" (Albuquerque, 1994). Asimismo, se observa también que si bien es importante considerar que las iniciativas de desarrollo local deben partir de los propios agentes de desarrollo local - es decir los próximos al territorio - hay iniciativas que pueden resultar de mayor potencialidad si son coordinadas con los planes y estrategias de desarrollo regional o nacional, tal como es el caso del PROFODE.

IV- Alburquerque (1994) postula también que el proceso de planificación tiene que ser visto como una herramienta para la implementación del enfoque del desarrollo endógeno.

En este sentido, las fases de la planificación del desarrollo económico local que propone el autor presentan similitud con las etapas que sigue el PROFODE (ver punto 1.2. Metodología), y que a continuación, de manera sintética, se pueden observar en el Cuadro $\mathrm{N}^{\circ} 2$. 


\section{Cuadro $\mathbf{N}^{\circ} 2$}

El Proceso de planificación

\begin{tabular}{|c|c|}
\hline $\begin{array}{c}\text { Fases de la planificación del desarrollo } \\
\text { económico local } \\
\text { (Albuquerque, 1994) }\end{array}$ & $\begin{array}{l}\text { Fases del PROFODE } \\
\text { (Sectur, Argentina, 2009) }\end{array}$ \\
\hline $\begin{array}{l}\text { - Recogida de información. Análisis y } \\
\text { diagnóstico. }\end{array}$ & -Etapa Preparatoria \\
\hline $\begin{array}{l}\text { - Definición de la estrategia de desarrollo } \\
\text { local: establecimiento de objetivos } \\
\text { generales y objetivos específicos. }\end{array}$ & \multirow[t]{2}{*}{-Etapa de Elaboración de Diagnóstico } \\
\hline $\begin{array}{l}\text { - Diseño de un plan de actuación e } \\
\text { identificación de los proyectos de desarrollo } \\
\text { local. }\end{array}$ & \\
\hline - Selección y gestión de proyectos. & -Etapa de Ejecución de Acciones Específicas \\
\hline - Ejecución de los proyectos. & \\
\hline - Control y evaluación. & -Monitoreo y evaluación de las distintas \\
\hline
\end{tabular}

Fuente: Elaboración propia

\section{REFLEXIONES FINALES}

En principio, se podría pensar que el PROFODE, como muchos otros programas implementados desde la administración pública nacional, y tal como lo establece Arocena (1995), corre el peligro de caer en "las formas centralistas de organización del territorio", como así también de profundizar la separación entre "planificadores y protagonistas". Sin embargo, a priori se puede percibir que en este programa existe una combinación de planificación e iniciativa interesante, por cuanto desde la administración central se promueve un cierto esquema de trabajo y ciertas etapas de acción, pero al mismo tiempo se parte de iniciativas locales y se identifican actores que actúan como nexo y facilitadores. Asimismo, se promueven metodologías participativas, se dejan instaladas capacidades según los requerimientos de cada sitio, y se fomentan, por ejemplo, ciertos microemprendimientos con base en el turismo, todo lo cual permite entrever que se trata 
de un programa encuadrado en la perspectiva del desarrollo endógeno, o que al menos está transitando ese camino.

Por otra parte, se podría decir que el programa encara una visión "arriba-abajo", propio de las estrategias centralizadas, y no viceversa "el desarrollo desde abajo" ó "abajo-arriba" para que realmente se constituya en un programa enmarcado en la visión del desarrollo endógeno. Sin embargo, y en coincidencia con Madoery $\left(2001_{b}\right.$ y 2003), se puede observar que en el PROFODE, si bien el Estado asume el rol de impulsor del desarrollo local, se reconocen las diversidades territoriales, y el desarrollo está ligado principalmente al esfuerzo de ciudades y regiones que tratan de impulsar iniciativas y de transitar su propia experiencia. Por lo tanto, a través de este programa, "el Estado puede recuperar su rol activo y positivo a través del diseño y de la implementación de políticas `desde abajo' (bottom up) que involucren a los agentes económicos y sociales territoriales, directa e indirectamente interesados en los procesos de desarrollo local y que son los reales destinatarios de las políticas” (Boscherini y Poma, 2000). Cabe aclarar, según estos últimos autores, que las acciones 'desde abajo' se caracterizan por una profunda interrelación entre estatal-privado, nacional-local, y requieren importantes procesos de cambio de los agentes y de las instituciones involucradas, apuntando a regenerar las relaciones en la sociedad civil, estimulando la participación de los agentes y de las instituciones políticas, económicas y sociales.

En síntesis, en el PROFODE, las políticas del gobierno central pueden verse fortalecidas con las políticas de cada sitio, a partir de la redefinición del Estado-Nación, el cual en su nuevo rol convive con otras escalas geográficas de interacción social, y tiene que articular con los factores de especificidad territorial. Es más, tal como plantea Madoery (2001 ), la articulación Estado-Región, es la clave del desarrollo regional, por lo que no hay desarrollo sostenido para una región, si no se enmarca dentro de una política nacional. Y al mismo tiempo, "ésta carece de toda viabilidad, si no se apoya en las capacidades endógenas de las regiones y si no muestra congruencia con la política económica general” (Boisier, 1998).

Es interesante destacar también que en la perspectiva del desarrollo endógeno según Arocena (1995) es importante la "multiplicación de las acciones locales en la renovación de actividades tradicionales..., la apertura de nuevos canales comerciales, la revitalización de la pequeña empresa”. En este sentido, los destinos turísticos emergentes promovidos por el PROFODE - y los pequeños y medianos emprendimientos que en consecuencia allí se generan - se alinean de alguna manera en esta tendencia. Asimismo, se podría confirmar que la puesta en valor o recuperación de ciertas localidades del interior del país, buscando en el turismo un nuevo elemento dinamizador de sus economías y generador de nuevos espacios y posibilidades para el desarrollo, va en 
coincidencia con lo que Albuquerque (2001) denomina "diversificación productiva como factor de solidez de la economía local”, y sigue la misma línea de lo que proponen Boscherini y Poma (2000), para quienes es preciso profundizar la posibilidad de fomentar el desarrollo de sistemas productivos locales ubicados fuera de los grandes centros urbanos, constituyendo un esquema de desarrollo alternativo para las economías locales.

En estas primeras reflexiones, se concluye que el PROFODE es un programa enmarcado en el enfoque del desarrollo endógeno porque promueve las iniciativas locales de desarrollo social, económico y ambiental con base en el turismo, brinda un marco institucional que partiendo de lo local encuentra su respaldo último en lo nacional, promueve el "emponderamiento" o empowerment, capacita los recursos humanos locales en base a las necesidades concretas del territorio, permite la construcción de redes, y estimula la participación del sector privado, entre otras acciones, todo en el marco de un proceso, que como define Vazquez Barquero (2000) permite que lo "local" se constituya en un espacio donde las iniciativas de los diversos actores de la sociedad organizada se vuelvan realidad, y donde lo social se integra con lo económico (Arocena, 1995).

Por otra parte, es de destacar que la iniciativa de ciertas localidades a participar del programa responde a una predisposición interna de sus propios actores que quieren - o al menos tienen fuertes expectativas - en constituirse en un posible destino turístico, fundamentado esto en la valorización de sus recursos endógenos, en la necesidad de diversificación de sus economías, y en la búsqueda de nuevas fuentes de empleo.

Por último, se observa que a través de los talleres participativos - donde además cabe destacar que se convoca a distintos sectores - se logra un acercamiento sobre las necesidades y demandas de la población local, y se construye a nivel social una "actitud favorable al desarrollo" a través del impulso de los procesos de participación ciudadana.

A partir, de esto, se podría aventurar que todo lo anterior permite en cada una de las localidades donde se implementa el PROFODE, liberar en las comunidades locales el potencial de creatividad e iniciativa necesarias para producir un verdadero proceso de desarrollo local, que sólo pueden construir los propios habitantes. En otras palabras, las comunidades de estos territorios comienzan a encontrar "a donde ir”...”, activándose así el "territorial institutional building" a nivel local (Boscherini y Poma, 2000), ya que se estimulan y generan ciertas condiciones que impulsan la creación de instituciones o 
espacios para el desarrollo, y se promueve un cambio cultural en los agentes locales, con el fin de impulsar la capacidad emprendedora y las acciones colectivas y consensuadas.

Todas estas consideraciones han tratado de establecer un puente, una conexión entre desarrollo endógeno y turismo, a través de un caso aplicado a la práctica. Queda aún un camino de análisis y de reflexión por recorrer, especialmente en lo que concierne a la aplicación concreta dentro de este programa de aspectos relativos a la creación de agencias de desarrollo local, el tratamiento de los eslabonamientos productivos o "clusters", la gestión y manejo de las fuentes de financiación y/u obtención de líneas de crédito, la promoción de inversiones, la disponibilidad y/o acceso a los recursos tecnológicos, aspectos todos incluidos y considerados ampliamente en el enfoque del desarrollo endógeno.

De todas maneras, como conclusión preliminar, podría decirse que el turismo entendido como una actividad diversificadora y dinamizadora del territorio, con base en los recursos propios y genuinos de cada localidad, puede convertirse - ya sea en una actividad económica complementaria, ya sea en una actividad económica principal - pero en cualquier caso, en uno de los motores factibles de promover un verdadero proceso de desarrollo local en estos "destinos emergentes", tanto desde la perspectiva de la eficiencia económica, del equilibrio ambiental, como de la equidad social, tal como lo plantea Cotorruelo Menta (2001).

Recibido: 25/07/2009

Aprobado: 17/11/2009

Arbitrado anonimamente 


\section{BIBLIOGRAFÍA Y FUENTES CONSULTADAS}

- Alburquerque, Francisco (1994). Metodología para el desarrollo económico local. En Manual de Desarrollo Local, editado por el gobierno vasco (Del Castillo, 1994), Cap. 28. http://www.redelaldia.org/article.php?id article=1114 (fecha de consulta: abril 2009)

- Alburquerque, Francisco (2001). La importancia del enfoque del desarrollo económico local. En A. Vázquez Barquero y O. Madoery. (eds.) Transformaciones globales y políticas de desarrollo local. Homo Sapiens ediciones, Rosario, Argentina.

- Arocena, José (1995). El desarrollo local: un desafío contemporáneo. Editorial Nueva Sociedad, Caracas.

- Boisier, Sergio (2004). Desarrollo Endógeno: ¿Para Qué?, ¿Para Quién? (El Humanismo en una Interpretación Contemporánea del Desarrollo), mimeo.

- Boisier, Sergio (2003). El desarrollo en su lugar (El territorio en la sociedad del conocimiento), mimeo, Santiago de Chile.

- Boisier, Sergio (2002). ¿Y si el desarrollo fuese una emergencia sistémica?, mimeo, Santiago de Chile.

- Boisier, Sergio (1998). Post-scriptum sobre desarrollo regional: modelos reales y modelos mentales. En Anales de Geografía de la Universidad Complutense, Madrid.

- Boscherini, Fabio; Poma, Lucio (2000). Más allá de los distritos industriales: el nuevo concepto de territorio en el marco de la economía global. En Boscherini, Fabio y Poma, Lucio (comp.). En Territorio, conocimiento y competitividad de las empresas. El rol de las instituciones en el espacio global, Miño y Dávila editores, Madrid.

- Cotorruelo Menta, Romeo (2001). Aspectos estratégicos del desarrollo local. En Vázquez Barquero, A. y Madoery, O. (comp.). En Transformaciones globales, Instituciones y Políticas de desarrollo local. Editorial Homo Sapiens, Rosario. http:// www.cedet.edu.ar/sitio/administracion/agenda/cotorruelo menta.pdf (fecha de consulta: mayo 2009)

- Madoery, Oscar (2008). Otro desarrollo. El cambio desde las ciudades y regiones. 1er Edición, San Martín UNSAM EDITA, Universidad Nacional de San Martín, Buenos Aires.

- Madoery, Oscar (2005). La "primera generación” de políticas locales de desarrollo en Argentina. Contexto, características y desafíos, mimeo, Buenos Aires.

- Madoery, Oscar (2001). El valor de la política de desarrollo local. En Vázquez Barquero, A. y Madoery, O. (comp.). Transformaciones globales, instituciones y políticas de desarrollo local, Homo Sapiens Ed., Rosario, 2001.

- Madoery, Oscar (2001 $)$. El Proyecto político local como alternativa de desarrollo. En Revista Política y Gestión, volumen 2, UNSAM, Homo Sapiens.

- Organización Internacional del Trabajo (OIT). (2009). Unidad Didáctica 2 “Desarrollo local y turismo: elementos político-institucionales claves”. Centro 
Internacional de Formación, Programa Delnet de Apoyo al Desarrollo Local, Curso de Turismo Sostenible y Desarrollo Local, Turín.

- Otero Urieta, Adriana (2007). La importancia de la visión de territorio para la construcción de desarrollo competitivo de los destinos turísticos. En Cuadernos de Turismo $\mathrm{N}^{\circ} 19$, Universidad de Murcia. http://revistas.um.es/turismo/article/ viewFile/13811/13331 (fecha de consulta: mayo 2009).

- Oyarzun, Edgardo; Szmulewicz, Pablo (1999). Fortalecimientos de la gestión en destinos turísticos. Fundamentos. En Revista Gestión Turística, No 3, Universidad Austral de Chile. http://www.gestionturistica.cl/publicaciones/revistas/anteriores/4. htm (fecha de consulta: julio 2009).

- SECTUR - Secretaría de Turismo de la Nación, Argentina (2005). Plan Federal Estratégico de Turismo Sustentable 2016. http://2016.turismo.gov.ar/wp turismo/ (Enlace: Institucional, PFETS - Plan Federal). (fecha de consulta: mayo 2009)

- SECTUR - Secretaría de Turismo de la Nación, Argentina (2009). Programa de Fortalecimiento y Estímulo a Destinos Turísticos emergentes. http://www.turismo. gov.ar/esp/menu.htm (Enlace: Institucional, Desarrollo Turístico, Destinos). (fecha de consulta: mayo 2009).

- SECTUR - Secretaría de Turismo de la Nación, Argentina (2008). Programa de Fortalecimiento y Estimulo a Destinos Turísticos emergentes. Ficha de postulación PROFODE - Convocatoria a Destinos 2008, (mimeo).

- SECTUR - Boletín Técnico - Plan Federal Estratégico de Turismo Sustentable 2016 (2007). "Red de organismos nacionales para el ordenamiento territorial”. Nota de prensa publicada el 07/10/2007. http://2016.turismo.gov.ar/wp_turismo/ (fecha de consulta: mayo 2009).

- SECTUR - Boletín Técnico - Plan Federal Estratégico de Turismo Sustentable 2016 (2007). "Diversificar la oferta para redistribuir la demanda". Nota de prensa publicada 09/07/2007 http://2016.turismo.gov.ar/wp turismo/?p=71 (fecha de consulta: junio 2009).

- SECTUR - Boletín Técnico - Plan Federal Estratégico de Turismo Sustentable 2016 (2008). "Nuevos destinos para el PROFODE". Nota de prensa publicada el 31/01/2008. http://2016.turismo.gov.ar/wp turismo/ (fecha de consulta: mayo 2009).

- Vázquez Barquero, Antonio (2001). Desarrollo endógeno y globalización. En Vázquez Barquero, A. y Madoery, O. (comp.). Transformaciones globales, instituciones y políticas de desarrollo local, Homo Sapiens Ed., Rosario.

- Vázquez Barquero, Antonio (2000). Desarrollo económico local y descentralización: una aproximación conceptual. CEPAL - GTZ, Santiago de Chile.

- Wallingre, Noemí (2007). Limitaciones o beneficios del desarrollo económico local en el turismo. En revista Tiempo de Gestión, III, Facultad de Ciencias de la Gestión, Universidad Autónoma de Entre Ríos. http://www.conocitur.com/ archivos/turismo-y-desarrollo/limitaciones-y-beneficios-del-desarrollo-local-en-elturismo-070601133950.pdf (fecha de consulta: mayo 2009). 